ribus ibi costae approximatis minoribus, ceteris margini approximatis longioribus, lateris paulo-angustioribus uniserialibus, costae approximatis, paucis, venis (praecipue inferioribus) subhorizontaliter-patentissimis. Spatha 5-pollicaris.

Nova-Granata (Caucana), Holton.

Monstera Pankemiana S. Folii lamina oblique-oblonga, apicem versus sensirn angustata, cuspidato-apiculata, basi latere latiore rotundata, latere conspicue angustiore arcuatocuneata, foraminibus interdum in latere latiore paucis, longulis, medianis iustructa, venis patentibus. Spatha.....

Demerara.

S c hön brun n, 9. Januar 1859.

\title{
Centsta mayeri.
}

\section{Von Vietor v. Janka.}

(Sect. Corniola M e d.) Perennis. Radix oblique breviterve descendens fibras agens paucas, graciles, longas, subcarnosas. Ca ale $s$, vel ex trunci annotini sublignescente residuo, terraeque accumbente rami: plures aut numerosi, her b a cei, stricti, tenues, sesquipedales circiter, tereti vel obsolete angulati, sulcatuli, inferne laeves glaberrimi, ceterum vero vel sparsissime adpresse pilosuli, vel apicem versus densiuscule puberuli v. pubescentes plerumque simplicissimi, rarius ramis in superiore parte aliquot, palulis (his fere semper sterilibus), brevibus, sparse foliatis ornati. Folia elliptica, late-ovalia vel rarius oblonga acuta; (inferiora saepe subrotunda) $19-22$ lin. longa, $14-10$ lin. lata, brevissime petiolata. Petioli ad summum 1/4 lin. longi, basi, vel paullo supra stipulati: stipulae subulatae, linea mox breviores, mox longiores. - Folia patentissima sunt; discoloria: supra enim dilute viridia, splendentia, convexa (numquam plana!); subtus pallidiora subopaca; margine piloso-pubescentia vel glabra, omnia apice mucronata. Inflorescentia simplicissima, pauciflora laxa: caulis saepissime a medio jam florifer. Folia bractealia ovato-lanceolata pedicellis (saltem inferiora) pluries longiores; immo infma legumina longe superant. Bracteae in caulis apice, ob legumina pleraque abortiva delapsa dense congestae, patulae v. reflexae, quam in $G$ en is ta tin ct or $i$ a longe majores, comam quasi haud raro formant. Pedicelli 1-2 lin. longi, demum indurati, crassiusculi, subclavati. In eorum apice bracteolae 2 , brevissimae, subulatae, rigidae. Calix sub $21 / 2$ lin. longus glaberrimus suboblique campanulatus: superiores dentes 2 inferioresque 3 subaequales, lanceolato-subulati, tubi longitudine, variantes breviores. Flores . . . . . . Legumina 9-17 lin. longa, linearia v. parum curvata plerumque patentis- 
sima, glaberrima, vel pilis sparsis accumbentibus adspersa; 5-10 sperma. Semina subrotunda, compressa, castanea, nitida.

Ha bit at.frequentissime ad viarum margines in silvis ad "Felix-Bad". prope Magno Varadinum Hungariae. Floret vere; legumina exeunte Augusto plane matura.

Stirpis tota facies distinctissima; - a Gonista tinctoria, iisdem fere locis, sed solitarie crescente toto coelo difert.-Dum haec flores fert, vel simul legumina juniora, illius fructus maturissimi. - A. G. ovata (G. nervata K it.), cui similior, quave floratione vernali convenit, glabritie omnium partium etc. valde recedit. Cum ceteris confundi non potest.

$\mathrm{Spach}$ lässt in seiner "Revisio generis Genista" in den annal. sciences naturell. ser. III. 3. (1845) pag. 121 et seq. der Sectio $V$ "Genistoides" drei Vnterabtheilungen. Sie zerfällt nämlich in:

Subdiv, a) Folia 1-nervia avenia, saltem obsolete et parce venulosa; fo- Rami novelli graciles, ratio vernalis. Subdiv. b) Folia 1-nervia magis minusve
venosa; floratio vernalis.

Subdiv, e) Rami novelli floriferi paniculato-vel racemoso-ramulosi. Floratio aestivalis. Folia saltem ramealia venose, modo 1-nervia modo subtriplinervia (variatione).

Zu a) gehören von europäischen Arten: Genista leptophylla $\mathrm{S}$ p a ch, die auch in Siebenbürgen sehr verbreitet ist, und der $\boldsymbol{G}$, triangularis sehr nahe steht. Ferner $\boldsymbol{G}$. depressa M. B. und $G$. tetragona Bess.

Zu b) $G$. anxantica T $\mathrm{T}$. und $G$. ovata W. et K. (von dieser letzteren Art ist $G$. nervata nach $K$ it a ib e l'schen Exemplaren, nicht verschieden).

Zu c) endlich: Genista lasiocarpa s p a ch $(G$. ovala Balb. flor. taurin., non W. et K.), zu welcher auch jene Pflanzen gehören, welche die Herren Schl os ser und Vuk otinovic bald als $G$. ovata, bald als $G_{+} n_{e r-}$ vata verschickten; dann $G$. tinctoria L., G. Delarbrei Lec. et Lam., $\boldsymbol{G}$, elata Wender. (G. elatior Ko ch) und $G$. mantica $\mathrm{P} 011$. - Die Species dies er Unterabtheilung bilden die Gattung Corniala M ed ic. bei P res l, mit Zuziehung der Genista triangularis W. und der G. ovata W. et $\mathbf{K}$.

Die von mir nun beschriebene $G$ en is $t$ a Mayer $i_{\text {, die ich dem }}$ verdienstvollen Herrn Dr. Med. A. M a y er in Grosswardein widme, welcher eben an einer ausfübrlichen Skizze der beiden Bäder daselbst, arbeitet, gehört zur Unterabtheilung b). Dass Genista ovata von meiner Art verschieden sei, erwähnle ich schon. Ich besitze vollkommene Exemplare in Blüthe und Frucht aus He uffe l's Hand, und habe die K i t a b el'sche Art Mitte Juli 1856 bei Mehadia mit reifer Frucht gesammelt. 
Von Genista anxantica habe ich ein Bruchstück, von Te no re selhst erhalten. Diese neapolitanische Art hat bedeutend kleinere und schmälere Blätter, und ist auch durch eine ganz andere Tracht, beinabe der von $G$. sagittalis, verschieden. - Genista Delarbrei schliesslich, hat ebenfalls breite, oberseits glänzende Blätter, aber den Habitus der gewöhnlichen G. tinctoria, weicht auch sonst von G. Mayeri im Bau der Schotten bedeutend ab und blüht im Sommer; man vergleiche hierüber Godron's und Grenier's "Flore de France" I. p. 353.

Ich bemerke nur noch, dass meine Art in der Natur als s ol che, aber da stets erkannt werden wird. - Es kostet keine Mühe die Pflanze sammt der schuhlangen Wurzel, die unter einer Menge abgefallenen Laubes von Eichen verborgen, kaum in den Boden einzuldringen scheint, zu erhalten.

In dieselbe Unterabtheilung stelle ich noch eine neue Art aus Siebenbürgen, die ich fälschlich als G. Iydia, auf den Heuwiesen bei Klausenburg gesammelt, im lahre 1855 versandte. Die wahre Genista lydia ist nach $\mathrm{Gr}$ i se b a ch im „iter hungaricum" mil G. leptophylla S pach einerlei, und kommt auf Kalkhergen häufig vor, hat aber mit der Klausenburger Pflanze, die ich G. campestris nenne, keino Aehnlichkeit. Diese werde ich beschreiben, sobald ich reife Früchte derselben gesehen, da ich nur mehr Blüthen-Exemplare vorliegend labe.

Wer sich mit den zahlreichen Genista-Arten abgegeben hat, wird zu beurtheilen wissen, wie schwierig sie zu unterschejden sind. Absolute Gültigkeit hat, einzeln genommen, fast kein Kennzeichen. Vielmehr sind alle vereint zu berücksichtigen, um Arten mit Sicherheit zu characterisiren.

W ien, am 95. December 1858.

\section{Beobachtungen in der Flora von Siebenbürgen,} nebst

Beschreibung neuer Pflanzenarten und Varietäten.

Von Dr. Ferd. Sehur.

II.

13. Agrostis L. gen. n. 80. Die siebenbürgischen Arten dieser Galtung lassen sich in zwei Sectionen eintheilen, von denen die eine jene Arten umfasst, deren Glumella zwei ausgebildete Paleen, die andern, deren Glumella nur eine vollkommene ausgebildete Palea besitzt. Die erste Section umfasst die Agrostes genuinae Euagrostis, Vilfa P. Be a u v., und wird characterisirt durch : Folia omnia plana, Glumella bipaleacea - die andern des Trichodium M i$\mathrm{c} \mathrm{h}$ a $\mathrm{u} \mathrm{x}$, wird characterisirt durch Folia difformia, infima et novellia angustiora, complicata vel setaceo-convoluta. Glumella, unipaleacea. 